\title{
Theileriosis (Cytauxzoonosis) in Roan antelope (Hippotragus equinus): Field exposure to infection and identification of potential vectors
}

\begin{abstract}
Authors:
Johan C.A. Steyl ${ }^{1}$

Leon Prozesky ${ }^{1}$

Wilhelm H. Stoltsz ${ }^{2}$

John A. Lawrence ${ }^{1}$

Affiliations:

${ }^{1}$ Department of Paraclinical Sciences, University of

Pretoria, South Africa

${ }^{2}$ Department of Veterinary Tropical Diseases, University of Pretoria, South Africa

Correspondence to:

Johan Steyl

Email:

johan.steyl@up.ac.za

Postal address

Private Bag X04,

Onderstepoort 0110,

South Africa

Dates:

Received: 02 Aug. 2011 Accepted: 13 Dec. 2011 Published: 20 Apr. 2012

How to cite this article: Steyl, J.C.A., Prozesky, L., Stoltsz, W.H. \& Lawrence, J.A., 2012, 'Theileriosis (Cytauxzoonosis) in Roan antelope (Hippotragus equinus): Field exposure to infection and identification of potential vectors', Onderstepoort Journal of Veterinary Research 79(1), Art. \#367, 8 pages. http:// dx.doi.org/10.4102/ojvr. v79i1.367
\end{abstract}

Four hand-reared, naïve roan antelope, 4 months of age, were exposed to naturally infected pasture on a game farm in Mpumalanga Province, South Africa, where roan are known to die from theileriosis. Various clinical parameters were recorded during this period. The predominant ticks parasitising these animals at the time (January to February), were Rhipicephalus appendiculatus and Rhipicephalus evertsi evertsi adults. After a period of 5 weeks the animals developed signs of clinical theileriosis and were treated with buparvaquone to prevent mortality. Primary hyperplasia of the local draining lymph nodes (Lnn. anorectales) near the feeding site of adult $R$. evertsi evertsi indicated possible transmission of Theileria sp. (sable) by this tick species. After recovery from theileriosis, these animals were confirmed carriers of Theileria sp. (sable) by PCR (polymerase chain reaction) and DNA probe analysis. Laboratory-bred larvae and nymphs of R. evertsi evertsi and R. appendiculatus respectively, were fed on the ears of these roan antelope. Salivary glands from moulted and prefed adult ticks of each species were dissected and stained for Theileria spp., and the PCR and DNA probe applied to a representative batch of dissected glands. $R$. appendiculatus adults collected from grass in infected camps were also dissected after prefeeding them on rabbits. Salivary glands of both tick species showed infected acini on staining and were also positive for Theileria sp. (sable) only, on multiprotozoal PCR-screening analysis. There was no statistical significant difference between the infection rate and the intensity of infection between the two tick species. $R$. appendiculatus ticks collected from grass were also PCR-positive for Theileria sp. (sable).

\section{Introduction}

Historically, a Theileria sp. (named Theileria hippotragi at the time) have been known to occur in roan antelope, Hippotragus equinus, in West Africa since 1912 (Todd \& Wolbach 1912) and in South Africa since 1974 (Wilson et al. 1974). A Theileria sp. also infects sable antelope (Hippotragus niger) in Southern Africa in regions where theileriosis occurs in roan (Neitz 1957; Wilson et al. 1974). Gene sequencing and comparison of isolates from recent cases in western roan (Hippotragus equinus koba) in Malelane (a town in the eastern parts of Mpumalanga Province in South Africa) confirmed, as had been suspected previously, that the Theileria sp. involved in Southern Africa is the same in roan and sable (Nijhof et al. 2005). It was originally isolated in lymphoblast cell culture from a sable that died of theileriosis in South Africa and referred to as Theileria sp. (sable) (Stoltsz \& Dunsterville 1992). Theileria sp. (sable) is certainly responsible for roan and sable mortalities in Mpumalanga and elsewhere in South Africa. This was supported by postmortal diagnosis of theileriosis in these species over a wide area of the northern and eastern parts of South Africa. Identification of the Theileria sp. involved, and confirmation of Theileria sp. (sable) as the pathogen, was performed by using a recently developed PCR (polymerase chain reaction) and DNA probe on reverse line blot (RLB) assay (Nijhof et al. 2005) for diagnostic purposes. Currently, no serological test exists that can be utilised in screening for Theileria sp. (sable) exposure or status. Opportunistic, randomly collected blood samples from various other species in the Malelane area tested positive for Theileria sp. (sable), (personal experience). These included nyala (Tragelaphus angasii), blue wildebeest (Connochaetes taurinus), reedbuck (Redunca arundinum), blesbok (Damaliscus pygarus phillipsi) and bushbuck (Tragelaphus scriptus). No mortality as a consequence of theileriosis has been described in these species.

Rhipicephalus appendiculatus (brown ear tick) is a well-known vector of Theileria sp. in Southern Africa (Norval, Perry \& Young 1992) and is also one of the more dominant species and widely distributed throughout the northern and eastern parts of South Africa. These factors have led to the suspicion that $R$. appendiculatus should be considered a strong contender as a vector for theileriosis in roan antelope. Early field observations, however, during outbreaks 
of the disease in roan, also supported the possibility of Rhipicephalus evertsi evertsi as a vector for Theileria sp. (sable).

The tick vector(s) for theileriosis in roan antelope has not been determined and therefore this study set out to establish the role of R. appendiculatus and R. evertsi evertsi as vectors for Theileria sp. (sable).

\section{Materials and methods Experimental design}

For this investigation, a farm in the summer rainfall region of South Africa known to suffer regularly from roan mortalities because of theileriosis, was selected in the Mpumalanga Province of South Africa. Diagnosis of mortality as a consequence of theileriosis was confirmed by the Section of Pathology, Faculty of Veterinary Science, University of Pretoria. The farmer, a breeder of endangered antelope species, was keeping the roan-breeding stock grouped in camps ranging in size from 20 ha to 30 ha.

Hand-reared roan calves known to be PCR-negative for Theileria sp. (sable) were exposed to ticks in a camp with a recent history of mortality as a result of theileriosis in roan antelope. Constant monitoring of various clinical parameters indicative of Theileria sp. infection was performed on these animals.

After recovery from clinical theileriosis, these calves were confirmed carriers of Theileria sp. (sable). Laboratory-bred immature stages $R$. appendiculatus and $R$. evertsi evertsi originating from cattle in the area were allowed to feed on these calves. After they moulted to adults, salivary gland dissections, staining, and PCR-screening for Theileria spp. and other blood parasites were performed.

\section{Hand-rearing of disease-free roan antelope calves}

Roan calves born during the 2002 calving season were handreared under tick-free conditions. Calves were collected from the pasture 24 hours after birth to ensure passive colostral immunity transfer and to prevent Theileria sp. infections from natural tick challenge (Shaw 2002). On arrival at the handrearing facility all calves were thoroughly dipped with a contact pyrethroid dip (Bayticol ${ }^{\circledR}$ ) and clearly identified by using plastic ear tags with a sequential numbering system.

There was no record of disease or mortality as a result of theileriosis during the period of hand-rearing of the roan antelope calves (44 calves in total). A few animals died from dietary and/or viral diarrhoea. The latter was mainly the result of Rotavirus infection as diagnosed by Lancet Laboratories during the first 6 weeks of age. Thereafter, there was no disease occurrence of any kind up to weaning at 7 months of age.

\section{Selection of trial animals and preparation for field tick exposure}

Four male roan calves (numbers 210, 218, 222 and 242), 4 months of age, were randomly selected. The animals were physically restrained and blood was collected in ethylenediaminetetraacetic acid (EDTA), stored at $-20{ }^{\circ} \mathrm{C}$, and submitted for PCR-testing as developed by Nijhof et al. (2005). A wide range of DNA-probes for various known Theileria, Babesia, Anaplasma and Ehrlichia species (Table 1), including controls and Theileria sp. (sable), was applied to the submitted samples (Nijhof et al. 2005). The blood samples collected from the four selected roan bull calves were all negative on PCR (RLB)-method, that is, the reverse line blot method) for all the haemoparasites, including Theileria sp. (sable).

On receipt of the PCR results, the four roan males were chemically immobilised by using a drug cocktail consisting of $20 \mu \mathrm{g} / \mathrm{kg}-35 \mu \mathrm{g} / \mathrm{kg}$ etorphine $\mathrm{HCl}$ and $0.3 \mathrm{mg} / \mathrm{kg}$ azaparone. They were translocated whilst under anaesthesia over a short distance to graze pastures in a camp on the farm that was known to be infected with theileriosis. Mortality in roan as a result of theileriosis had occurred in this camp no longer than 6 months prior to this experiment, and it had since been accommodating a roan herd. This herd was transferred to an adjacent camp 2 weeks prior to the experiment. The four males were purposely left undipped in an attempt to expose them to ticks as soon as possible after arrival. This field trial was conducted during January and February 2003, coinciding with the tick activity season and tick numbers were close to a peak.

\section{Monitoring of Theileria infection}

The animals were still being bottle-fed and as a result it proved very easy to habituate them to handling, thus facilitating a wide spectrum of possible monitoring parameters. Several parameters were monitored over a period of 6 weeks. The time to noticeable tick infestation, species present, attachment sites and subjective assessment of tick load were recorded. Rectal temperature was measured twice daily in the morning and afternoon at feeding times with a calibrated digital thermometer. A rectal temperature

TABLE 1: List of haemoparasites screened for by polymerase chain reaction, reverse line blot method.

\begin{tabular}{lll}
\hline $\begin{array}{l}\text { Ehrlichia/Anaplasma } \\
\text { catch all }\end{array}$ & $\begin{array}{l}\text { Theileria/Babesia } \\
\text { catch all }\end{array}$ & $\begin{array}{l}\text { Theileria spp. } \\
\text { catch all }\end{array}$ \\
\hline A. centrale & B. catch all 1 & T. sp. kudu \\
A. marginale & B. catch all 2 & T. sp. (sable) \\
A. phago & B. felis & T. bicornis \\
E. ruminantium & B. divergens & T. annulata \\
A. bovis & B. microti & T. buffeli \\
E. chaffiensis & B. bigemina & T. sp. buffalo \\
E. sp. omajienne & B. bovis & T. mutans \\
E. canis & B. rossi & T. parva \\
- & B. canis & T. taurotragi \\
- & B. vogeli & T. velifera \\
- & B. major & T. equi \\
- & B. bicornis & T. lestoquardi \\
- & B. caballi & T. annae \\
- & B. gibsoni & - \\
- & B. sable & - \\
\hline - & B. leo & - \\
\hline - & B. occultans & - \\
\hline
\end{tabular}


higher than $40^{\circ} \mathrm{C}$, especially if the ambient temperature was below $28^{\circ} \mathrm{C}$, was considered a fever reaction. Subjective scoring values of 1-4 were applied to assess feeding behaviour, with 4 indicating good general health and behaviour progressively deteriorating to 1 , which implied clinical illness. The peripheral lymph nodes were palpated to assess swelling. Scoring of lymph node size (0-4) with 4 being visibly enlarged without palpation, and 0 normal on palpation, was given. Fine-needle aspiration, using a sterile $1 \frac{1}{2}$ " 20G hypodermic needle, was simultaneously performed on the superficial cervical and other lymph nodes if they were visibly enlarged at times of blood collection. Thin smears were prepared as described for peripheral blood smear staining and examination. Peripheral blood smears were obtained every second day by pricking of the tail tip with a $\frac{3}{4} " 21 G$ needle, fixed and stained by using the Diff Quik $^{\circledR}$ staining method. The sample was immediately examined with a standard light microscope for lymphoblastic transformation and theilerial schizont and/or piroplasm presence. Blood smears were examined for other vectorborne haemoparasitic infections such as Anaplasma spp. and Babesia spp. Blood was also collected in EDTA every 7 days during chemical immobilisation and kept at $-20{ }^{\circ} \mathrm{C}$ freezer for later PCR-testing. Clinical observation included examination of the ocular conjunctival mucosae and the colour was subjectively assessed at regular intervals.

\section{Treatment of clinical theileriosis}

A clinical case of theileriosis was defined by using the following criteria: pyrexia above $40{ }^{\circ} \mathrm{C}$ shortly followed by, preceded or accompanied by, theilerial schizonts in circulation detectable by blood smear examination. Sick animals received anti-theilerial therapy by using buparvaquone (Butalex ${ }^{\circledR}$ ) at $2.5 \mathrm{mg} / \mathrm{kg}$ intramuscularly once. If the fever did not abate 48 hours later, a second injection was administered. A staggered treatment regimen was applied to the different animals in an attempt to determine the efficacy of anti-theilerial therapy in relation to the first day of fever. The following regimen was applied: Animal 218 was treated on the first day of pyrexia. Animal 210 was treated one day, Animal 222 two days, and Animal 242 three days, post-pyrexia.

\section{Tick feeding on Theileria sp. (sable) carrier animals}

Clean ticks for Theileria pick-up experiments were obtained from engorged females of $R$. evertsi evertsi and $R$. appendiculatus, originating from cattle in the Malelane area and transferred to the laboratory for egg laying by using standard operating procedures (Heyne, Elliott \& Bezuidenhout 1987). Larvae of R. appendiculatus (3-host tick) were fed on rabbit ears and backs after hatching and left to engorge. These engorged larvae were collected and stored in batches of 250 in ventilated plastic vials to moult and mature to nymphae under controlled laboratory conditions. Eggs from $R$. evertsi evertsi (2-host tick) were weighed and divided into batches that contained an estimated 500 eggs each. These eggs were left to hatch and mature before the onset of the tick feeding trial.

Upon PCR confirmation of carrier status for Theileria sp. (sable), the four animals were relocated to tick-free boma conditions. They were prepared for tick feeding by applying linen ear-bags to their ears (Heyne et al. 1987). Two modifications were made to the described method. A head bandage was applied with adhesive tape (Elastoplast ${ }^{\circledR}$ ) and temporary nylon skin sutures were strategically placed to prevent animals from shaking loose the ear-bags. The animals were chemically immobilised to fit the ear-bags safely and to facilitate the placement of ticks.

The four animals were divided into pairs. One pair received $R$. evertsi evertsi larvae and the other received nymphae of R. appendiculatus, which were applied randomly in batches of 500 per ear per animal.

After 6 days, the animals infested with the R. appendiculatus nymphae were immobilised and the bags with engorged nymphae removed. It was necessary to scrape off engorged, but still attached, nymphae by using the shaft of a scalpel handle. The ear-bags of the animals containing $R$. evertsi evertsi larvae were removed after 14 days and engorged nymphae collected.

\section{Tick salivary glands dissection, staining and polymerase chain reaction-testing}

The engorged nymphae of both tick species were kept under standard laboratory conditions until moulted to adults (Heyne et al. 1987). The ensuing adult ticks were then fed on laboratory rabbits for a period of 4 days to allow salivary gland maturation to occur (Morzaria et al. 1999; World Organisation for Animal Health 2008). The rabbits were euthanised with sodium pentobarbitone (Eutha-Naze ${ }^{\circledR}$ ) and ticks were removed immediately with curved forceps, taking care not to put pressure on the anterior region of the attached ticks. These ticks were placed in a tick-proof ventilated container and, within 4 hours, individually embedded with the scutum facing up in a Petri dish containing paraffin wax. Ticks, 100 of $R$. evertsi evertsi and 150 of $R$. appendiculatus, of equal numbers of male and female ticks, were then dissected in phosphate buffered saline (PBS) by using a standard stereomicroscope. Salivary glands were removed and transferred to a glass microscope slide to dry. The slides were then processed by staining with methyl-green and pyronin (Walker et al. 1979) and examined for Theileria spp. infected salivary acini with a standard light microscope.

A representative sample of about 50 ticks for each species was dissected, the salivary glands removed and suspended in $2 \mathrm{~mL}$ vials containing an isotonic saline solution for PCR detection of Theileria sp. (sable) and possible other protozoal organisms. A screening profile of a wide range of vectorborne haemoparasites including Theileria spp., Anaplasma spp., Ehrlichia spp., Babesia spp., and others (Table 1) was applied to these samples using the RLB method. 


\section{Field ticks}

Approximately 160 adult $R$. appendiculatus ticks collected by hand from grass stalks during the February from various roan-grazing camps on the farm, were allowed to feed on rabbits for 4 days and were included as a separate sample. These camps experienced mortalities as a result of theileriosis about 6 months earlier, 2 months into the roancalving season. A total of 100 ticks were dissected and stained as above. Additional salivary glands from 30 ticks were submitted for PCR.

\section{Results}

\section{Monitoring of Theileria infection} Tick infestation

Larval infestation occurred only after about 2 days and adult infestation 5 days after introduction of the roan into the camp. This was marked by increased scratching activity, especially of the ears. The adult stage of $R$. evertsi evertsi feeding in perianal and inguinal regions was first noted. Their numbers never increased to more than 20 per animal. Rhipicephalus evertsi evertsi immatures (larvae and nymphae) were present in low numbers in the outer ear canal. Secondly, $R$. appendiculatus adults started feeding around the same time but occupied other regions involving the head, neck and outer surface of the ear pinna. Their numbers were higher than $R$. evertsi evertsi, ranging from approximately 40 to 80 at a time per animal. Immatures were also present in low numbers on the inner peripheral area of the ear pinna.

Occasionally other tick species could be found, either singly or in very low numbers of less than five. These included Rhipicephalus (Boophilus) species around the neck and shoulder, and Amblyomma hebraeum in the inguinal and perianal region.

\section{Rectal temperature}

Rectal temperatures recorded in the afternoons were found on average to be higher than morning temperatures and as such were not considered. Morning rectal temperatures were indicative of a true pyrexia (Figures $2-5$ ). The earliest pyrexic reaction (higher or equal to $40^{\circ} \mathrm{C}$ ) was observed in Animal 218 at 34 days post-release into the infected camp. The days to first pyrexia for the other animals were as follows: Animal 210 on day 36, Animal 222 on day 37, and Animal 242 on day 39. The fever reactions in the animals had specific temporal relationships with blood smear and lymph node size changes (Figures 2-5). Rectal temperatures remained high for a day or two after anti-theilerial therapy.

\section{Habitus}

During the first 2 weeks of exposure the animals showed slight tick worry but soon became habituated. All animals were feeding well and continuously scored 4 until the day of pyrexia. Although still drinking milk, enthusiasm decreased and all animals lost their appetite within 24 hours after the first pyrexia. All scored around 1 to 2 at this stage. The duration of poor habitus was longer for Animals 222 and 242 and continued whilst their rectal temperatures remained high. Within $24 \mathrm{~h}$ after the rectal temperatures dropped to within the normal range, habitus steadily improved over 3 days. Grazing activity was poor for all animals during the first 10 days after treatment for theileriosis. Approximately 2 weeks after treatment for theileriosis, all animals showed a marked loss in body condition, which relates to poor habitus during recovery from the infection.

\section{Peripheral lymph node palpation}

The superficial cervical lymph node (SLn) was the easiest to palpate and score. A gradual increase in size of all four animals' SLn could be detected, scoring around 2 from 2 weeks after field introduction. Five days prior to the first animal (218) showing pyrexia, the anorectal lymph nodes (ALn), bilateral to the tail base, were visibly enlarged
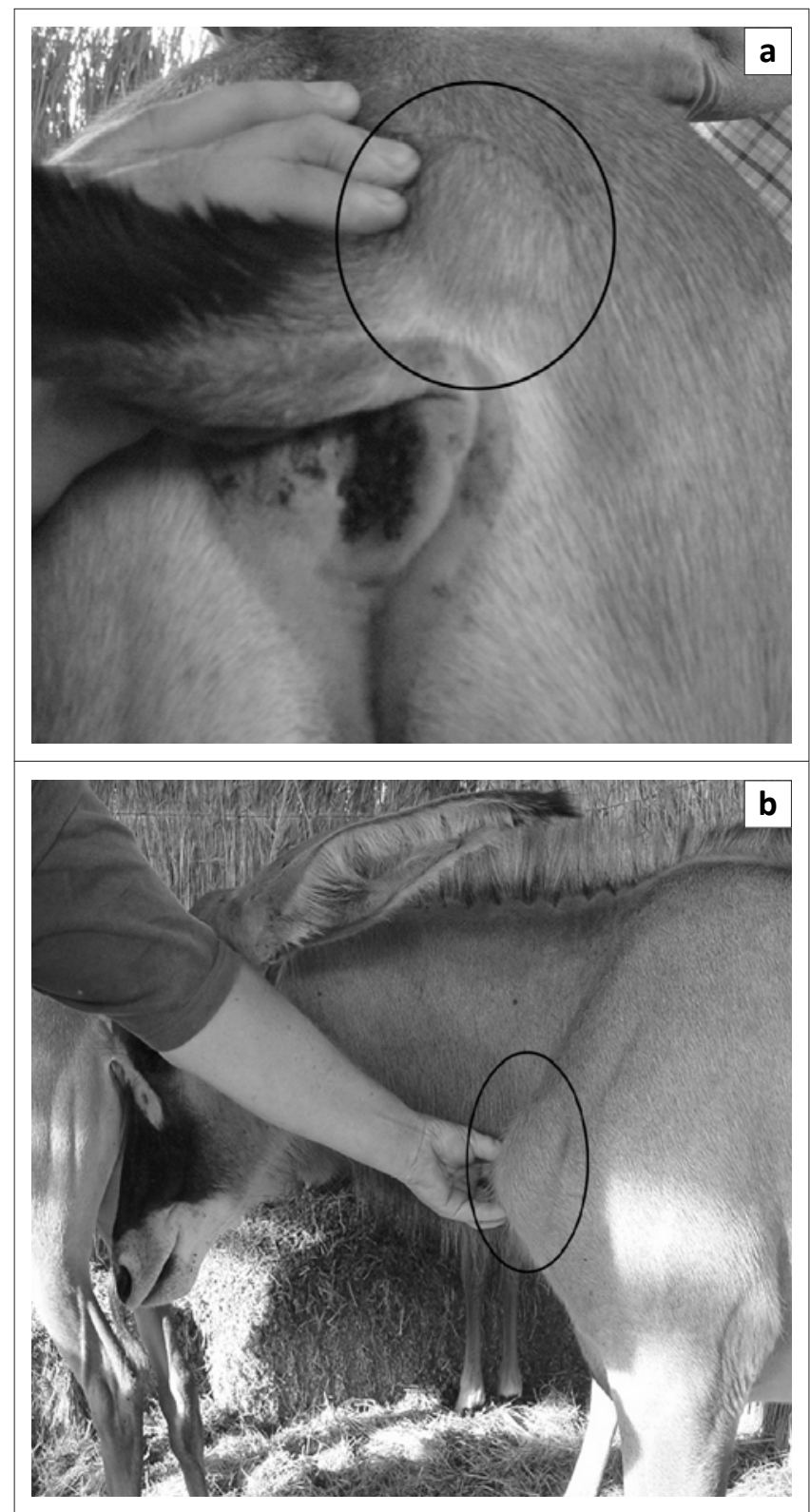

Score: 4.

FIGURE 1: Marked (a) anorectal and (b) superficial cervical lymphadenomegaly. 
(score 4), (Figure 1a). At the time of first pyrexia, a marked general enlargement of peripheral lymph nodes could be detected, scoring between 3 and 4 (Figure 1b). This temporal pattern was the same for all four animals. Within 7 days after treatment for theileriosis, the lymph node size decreased considerably and reverted to a score of 2 , with the anorectal nodes becoming visibly and palpably undetectable 10 days after treatment.

\section{Lymph node aspiration}

At introduction of the roan to tick-infested veld, lymph node aspirates showed predominantly small lymphocytes, with few blast-transformed lymphocytes and an occasional macrophage. At first enlargement of the SLn. (score 2) there was an increase in the blast-transformed lymphocyte complement and also occasional eosinophils. This finding continued until the first anorectal lymph node impression smear was performed in reaction to marked enlargement (score 4 on Animal 218, 5 days prior to pyrexia). At this stage, no equivalent enlargement could be detected in the SLn's or in any other lympho-centres. Initially, on cytology of ALn aspiration, blast-transformed lymphocytes predominated, some of which were atypical and appeared neoplastic. The cytological features in these cells were large, round to ovoid, often indented to bean-shaped nuclei, containing one or two large nucleoli and increased amounts of amorphous

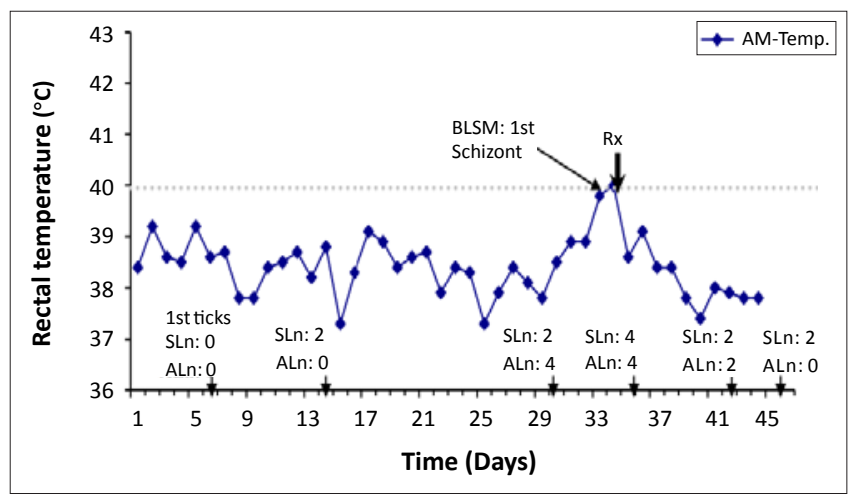

BLSM, blood smear; SLn, superficial cervical lymph node score (0-4); ALn, anorectal lymph node score (0-4); Rx, buparvaquone treatment.

FIGURE 2: Animal 218: Blood smear findings, lymph node findings and day of treatment in relation to rectal temperature over time.

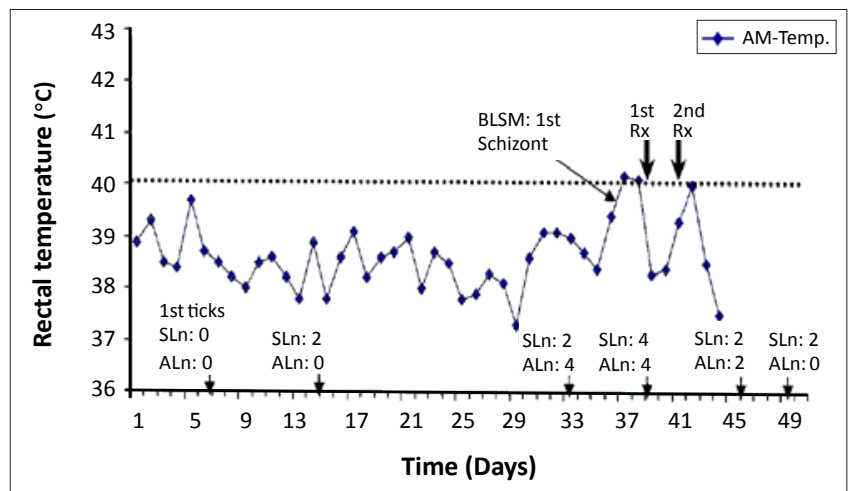

BLSM, blood smear; SLn, superficial cervical lymph node score (0-4); ALn, anorectal lymph node score (0-4); Rx, buparvaquone treatment.

FIGURE 4: Animal 222: Blood smear findings, lymph node findings and day of treatment in relation to rectal temperature over time. basophilic cytoplasm. Regular mitotic figures also featured. It sometimes proved difficult to differentiate between these 'neoplastic' lymphoblasts and immature monocytes. No typical theilerial schizonts (Koch's bodies) could be detected at this stage on the ALn impression smears. At the time of pyrexia, all peripheral lymph nodes scored 4 and impression smears showed marked lymphocytic blast transformation, in addition to cytoplasmic protozoal inclusion bodies characteristic of theilerial schizonts. Within 1 week of treatment for theileriosis, the impression smears were characterised by decreased lymphoblast numbers, large numbers of pyknotic and karyorrhexic lymphocytes and nuclear debris in the background. Theilerial schizonts were difficult to find from $72 \mathrm{~h}$ post-buparvaquone therapy and when present, showed marked pyknosis.

Similar lymph node changes and cytological findings could be demonstrated in the other animals at the time of their respective reactions.

\section{Peripheral blood smears}

In comparison to blood smears performed prior to field exposure, peripheral smears showed increased numbers of eosinophils from about 1 week into the trial that coincided with slight lymph node enlargement (score 2). From 3-5 days prior to pyrexia, a progressive atypical lymphoblastosis could

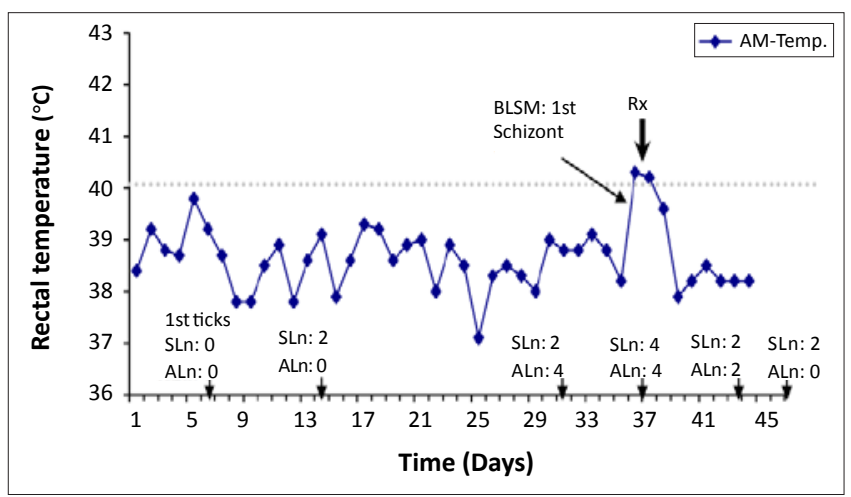

BLSM, blood smear; SLn, superficial cervical lymph node score (0-4); ALn, anorectal lymph node score (0-4); Rx, buparvaquone treatment.

FIGURE 3: Animal 210: Blood smear findings, lymph node findings and day of treatment in relation to rectal temperature over time.

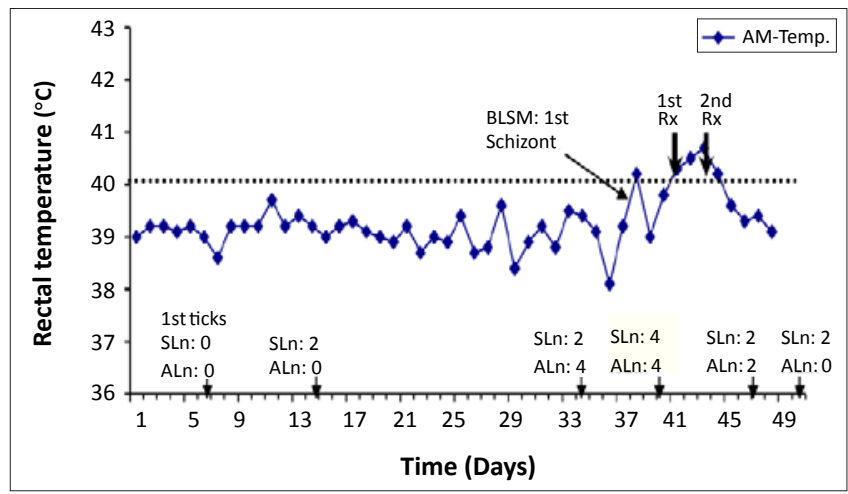

BLSM, blood smear; SLn, superficial cervical lymph node score (0-4); ALn, anorectal lymph node score (0-4); Rx, buparvaquone treatment.

FIGURE 5: Animal 242: Blood smear findings, lymph node findings and day of treatment in relation to rectal temperature over time. 
be detected. This change continued, with the first cytoplasmic theilerial schizonts demonstrable in lymphoblasts 24 hours prior to the first fever reaction. The first intra-erythrocytic theilerial piroplasms could be seen from the day of fever, with numbers increasing as the disease progressed. As a result of antitheilerial therapy at the time of pyrexia, the numbers of theilerial schizonts and piroplasms decreased over the following 4-6 days until only the occasional piroplasm could be detected. Examination for other microscopically detectable haemoparasites was negative.

\section{Mucous membrane examination}

The mucous membrane colour was pink at the start of the trial and remained so until around 5 days after the first fever reaction. The mucous membrane colour at this stage varied from pale pink at first, progressively turning pale yellow around 2 days later. Animal 242 showed more intensely pale yellow mucous membranes in comparison to the others. The normal pink mucous membrane colour returned within 2 weeks after treatment.

\section{Blood samples for polymerase chain reaction detection of Theileria sp. (sable)}

Polymerase chain reaction results showed that all four animals were negative prior to field exposure. They became positive for Theileria sp. (sable), coinciding with the first fever reaction and remained as such 6 weeks later.

\section{Treatment of clinical theileriosis}

Animals responded well to treatment with buparvaquone with clinical parameters improving and returning to normal within the expected time range for each (see above). Animals 222 and 242, which received delayed treatments, 2 and 3 days respectively after the first pyrexia, required a second injection of buparvaquone because their rectal temperatures remained high for longer than 48 hours post-treatment. The other two animals showed a significant drop in rectal temperature within 48 hours, and did not require a second injection of buparvaquone.

\section{Tick feeding on Theileria sp. (sable) carrier animals}

Tick yields from individual animals varied considerably depending on the species. From a total of 2000 R. evertsi evertsi larvae applied to ear-bags in two animals, only about 380 viable engorged nymphae could be recovered. In comparison, the number of $R$. appendiculatus nymphs, which dropped engorged, was about 590 in total (Figures 6a and $6 b)$.

\section{Salivary gland investigation of prefed ticks}

Feeding ticks on rabbits was very successful, with more than $90 \%$ of placed ticks attaching after 12 hours, which resulted in high recovery rates of ticks after 4 days of feeding. Methylgreen and pyronin tick salivary gland staining was typical, as described by Walker et al. (1979), (Figure 7). The quantitative results are illustrated (Table 2 ).
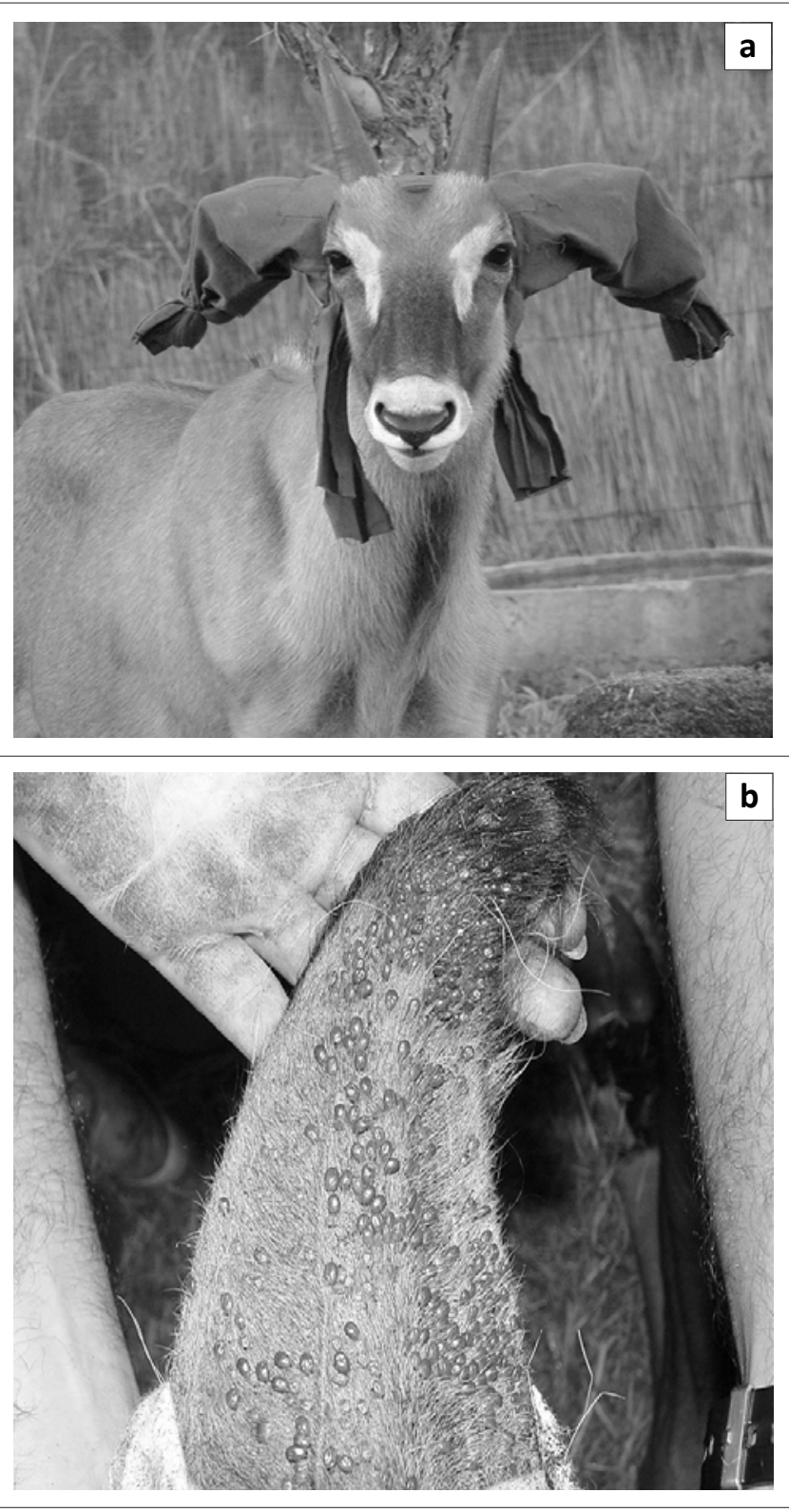

FIGURE 6: (a) Calico cloth ear-bags protecting Rhipicephalus appendiculatus nymphae feeding on a roan antelope calf and (b) Engorged Rhipicephalus appendiculatus nymphae ready to be collected.

TABLE 2: Infection rate with Theileria in ticks recovered from carrier roan

\begin{tabular}{lll}
\hline Assessed parameters & $\begin{array}{l}\text { Rhipicephalus } \\
\text { evertsi evertsi }\end{array}$ & $\begin{array}{l}\text { Rhipicephalus } \\
\text { appendiculatus }\end{array}$ \\
\hline Total ticks dissected & 100 & 150 \\
Total infected ticks & $5(5 \%)$ & $5(3.3 \%)$ \\
Infected salivary acini & 9 & 6 \\
Average infected acini per infected tick & 1.8 & 1.2 \\
\hline
\end{tabular}

TABLE 3: Methyl-green and pyronin tick salivary gland staining and polymerase chain reaction. Infection rate in adult Rhipicephalus appendiculatus ticks collected from grass.

\begin{tabular}{ll}
\hline Assessed parameters & Results \\
\hline Total dissected & 100 \\
Total infected ticks & $9(9 \%)$ \\
Infected salivary acini & $42(11) \dagger$ \\
Average infected acini per infected tick & $4.6(1.2) \dagger$ \\
PCR (RLB) for Theileria sp. (sable) (30 ticks) & Positive \\
\hline
\end{tabular}

$\mathrm{PCR}$, polymerase chain reaction; $\mathrm{RLB}$, reverse line blot.

$\dagger$, One tick had 31 infected acini (the value in brackets indicates number excluding this tick). 
The PCR (RLB) results for 50 dissected salivary glands from $R$. evertsi evertsi and R. appendiculatus were positive for Theileria sp. (sable) in both species.

\section{Field ticks}

The infection rate in adult $R$. appendiculatus collected from grass, is shown (Table 3).

\section{Discussion}

There has been no attempt made previously to identify the vector of theileriosis in roan, but field observations that outbreaks usually coincided with heavy infestations with $R$. appendiculatus, led to the assumption that this species was a potential vector. Rhipicephalus evertsi evertsi has been viewed as an apparently incidental parasite, but was included in this investigation as it is known to transmit other Theileria sp.

In ticks fed experimentally on carrier animals, no statistically significant difference in infection rate per infected tick or the number of ticks infected with Theileria sp. (sable) could be demonstrated between $R$. evertsi evertsi and $R$. appendiculatus. From these and PCR findings, it can be concluded that both species become infected with Theileria sp. (sable) and are potential vectors. Primary anorectal hyperplastic or 'neoplastic' lymphadenomegaly was a very important finding in support of identifying the main vector for theileriosis in roan exposed to natural tick infection. It is well known from theilerial kinetic studies, that the local draining lymph node(s) is the first to enlarge during the initial stages of theileriosis in cattle (Morrison et al.1981).The drainage area for the anorectal lymph node includes the perianal region and tail. The only ticks feeding at this site at the time of lymphoid proliferation were $R$. evertsi evertsi adults, suggesting that $R$. evertsi evertsi was more likely to be the transmitting vector than $R$. appendiculatus in these cases. This, however, does not exclude $R$. appendiculatus as a vector, as PCR results on dissected salivary glands showed that it was also infected with Theileria sp. (sable).

As a consequence of the need for chemical restraint to perform lymph node aspirates, daily lymph node aspiration to determine the temporal relationship of primary nodal (ln. anorectales) theilerial schizont appearance, versus the first appearance in other lymph nodes and the peripheral blood, was not possible.

During tick salivary gland dissections, one field-collected $R$. appendiculatus tick exhibited a very high infection with Theileria. This infection could not be confirmed to be Theileria sp. (sable), and comment on its significance would be speculative. If it is assumed that this infection was Theileria sp. (sable), it would indicate that $R$. appendiculatus ticks are capable of acquiring heavy infection rates, supporting its role as a vector significantly.

Tick yields from ears were very low for $R$. evertsi evertsi in comparison with $R$. appendiculatus. This could be ascribed to

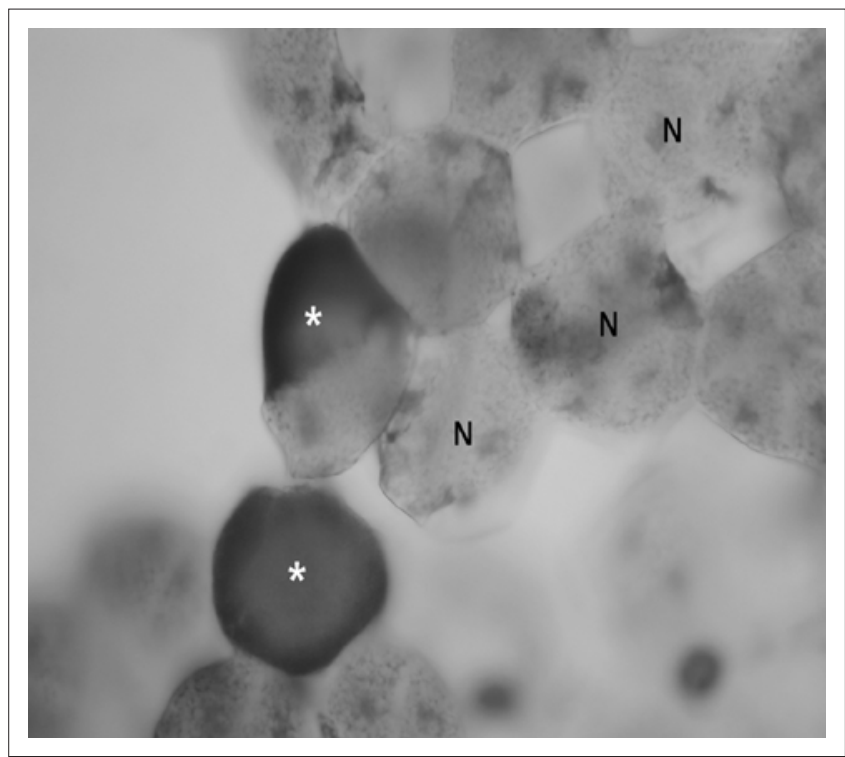

*, Infected tick salivary acini staining finely granular and intensely basophilic; $\mathrm{N}$, normal salivary acini.

FIGURE 7: Methyl-green and pyronin stain.

prolonged housing in ear-bags and exposure to host-inflicted trauma from head-shaking and scratching.

Based on the rectal temperature results, it would be difficult at this stage to be sure about the incubation period of theileriosis in roan, as it was not possible to determine the day of infection by infected ticks under field conditions. At best, one can state that it appears to be less than 33 days (earliest temperature reaction) in most cases. An infection trial, possibly with an infected tick-derived stabilate or known infected live ticks, would be necessary to settle this issue.

The use of buparvaquone as anti-theilerial chemotherapy is highly effective against theileriosis in roan antelope, but the staggered delay in treatment in relation to the first day of pyrexia resulted in Animals 222 and 242 (treatment delay of 2 and 3 days respectively) requiring a second injection of buparvaquone. This finding revealed the importance of administering buparvaquone as close as possible to the initial fever reaction. A delay of anti-theilerial therapy may result in more serious complications and death, even if treated with a very effective anti-theilerial drug such as buparvaquone.

No animal in this trial needed supportive therapy for opportunistic infections such as pneumonia that is known to be a complication in cattle recovering from East Coast fever (Muraguri, Kiara \& McHardy 1999; Wagner et al. 1975).

During the first 2 weeks of field tick exposure, a small increase in size of superficial cervical lymph nodes could be palpated and peripheral blood smear eosinophilia was detected. These changes were ascribed to expected hyperplasia [see SLn fine-needle aspiration (FNA) impressions smears] and inflammation as a result of ectoparasitic (mainly ticks) dermatitis. 
Post-pyrexic changes in the mucous membrane colour of the animals were indicative of anaemia: (pale pink), later becoming complicated by icterus (pale yellow). These changes are consistent with signs occasionally observed in cattle suffering from East Coast fever (Theileria parva), (Lawrence, De Vos \& Irvin 1994), or Theileria annulata infection (Pipano \& Shkap 2004).

Loss of body condition during the post-therapeutic recovery phase was mostly likely a consequence of less time spent grazing with more time spent lying down and resting, although the animal would still drink milk reluctantly. Anaemia and systemic inflammation also accounted for additional nutritional demands on the animal.

It was the first time that theileriosis, which has been well studied in cattle, has been followed clinically in detail under field conditions in a wild antelope species. Previously, limited observations were recorded with experimental and natural Theileria taurotragi infection in eland antelope (Grootenhuis et al. 1980). Careful monitoring of various clinical parameters revealed many similarities in the clinical development and progression of theileriosis (especially East Coast fever) in cattle and in roan antelope.

\section{Conclusion}

Clinical theileriosis in roan antelope responds favourably to buparvaquone treatment, provided that it is administered as close as possible to the primary pyrexic phase. For more exact pathogenesis studies, parasite transmission and disease monitoring will have to be repeated in a controlled environment with known infection times and dose rates.

Valuable information gathered during field tick exposure demonstrated that $R$. evertsi evertsi and $R$. appendiculatus are two potential vectors of theileriosis in roan antelope. To determine the role of both species in the epidemiology of theileriosis outbreaks in roan, tick infection and transmission trials that involve carrier versus piroplasmaemic hosts should be compared separately for both tick species.

\section{Acknowledgements}

Our sincere appreciation for the financial, infrastructural, and personnel support provided by Mr J. Hume, owner of Mauricedale Game Ranch. A special acknowledgement of gratitude to Mrs Anita York, without whose dedication and love for these animals this project may never have reached its full potential. Polymerase chain reaction tests were performed by the molecular laboratory in the Department of Veterinary Tropical Diseases, University of Pretoria. The research team also acknowledges the significant funding contribution from The Department of Trade and Industry of South Africa through the Technology and Human Resources for Industry Programme (THRIP).

\section{Competing interests}

The authors declare that they have no financial or personal relationship(s) which may have inappropriately influenced them in writing this paper.

\section{Authors' contributions}

L.P. (University of Pretoria) was the project leader. J.C.A.S. (University of Pretoria) and J.A.L. (University of Pretoria) were responsible for the experimental and project design. J.C.A.S. (University of Pretoria) performed all the experiments. W.H.S. (University of Pretoria) made conceptual contributions. J.C.A.S. (University of Pretoria) wrote the manuscript and J.A.L. (University of Pretoria) edited the language and grammar. Blood sample testing was performed by the molecular biology laboratory in the Department of Veterinary Tropical Diseases, University of Pretoria.

\section{References}

Grootenhuis, J.G., Morrison, W.I., Karstad, L., Sayer, P.D., Young, A.S., Murray, M. et al., 1980, 'Fatal theileriosis in eland (Taurotragus oryx): Pathology of natural and experimental cases', Research in Veterinary Science 29, 219-229. PMid:7193340

Heyne, H., Elliott, E.G.R. \& Bezuidenhout, J.D., 1987, 'Rearing and infection techniques for Amblyomma species to be used in heartwater transmission experiments', Onderstepoort Journal of Veterinary Research 54, 461-471. PMid:3448576

Lawrence, J.A., De Vos, A.J. \& Irvin, A.D., 1994, 'East Coast fever', in J.A.W. Coetzer G.R. Thomson \& R.C. Tustin (eds.), Infectious diseases of livestock with special reference to Southern Africa, pp. 309-325, Oxord University Press, Cape Town.

Morrison, W.I., Buscher, G., Murray, M., Emery, D.L., Masake, R.A., Cook, R.H. et al., 1981, 'Theileria parva: Kinetics of infection in the lymphoid system of cattle', Experimental Parasitology 52, 248-260. http://dx.doi.org/10.1016/00144894(81)90080-1

Morzaria, S.P., Spooner, P., Bishop, R. \& Mwaura, S., 1999, 'The preparation of a composite stabilate for the immunisation against East Coast fever', in S. Morzaria \& S. Williamson (eds.), Proceedings of an FAO/ILRI/OAU-IBAR workshop, Nairobi, Kenya, 10-12 March 1997, pp. 56-61.

Muraguri, G.R., Kiara, H.K. \& McHardy, N., 1999, 'Treatment of East Coast fever: A comparison of Parvaquone and Buparvaquone', Veterinary Parasitology 87, 2537. http://dx.doi.org/10.1016/S0304-4017(99)00154-5

Neitz, W.O., 1957, 'Theileriosis, gonderiosis and cytauxzoonoses', Onderstepoort Journal of Veterinary Research 27, 275-429.

Nijhof, A.M., Pillay, V., Steyl, J., Prozesky, L., Stoltsz, W.H., Lawrence, J.A. et al., 2005, 'Molecular characterization of Theileria species associated with mortality in four species of African antelopes', Journal of Clinical Microbiology 43, 5907-5911. http://dx.doi.org/10.1128/JCM.43.12.5907-5911.2005, PMid:16333074

Norval, R.A.I., Perry, B.D. \& Young, A.S., 1992, The epidemiology of theileriosis in Africa, Academic Press, London.

Pipano, E. \& Shkap, V., 2004, Theileria annulata theileriosis, Oxford University Press, Oxford.

Shaw, M.K., 2002, 'Theileria development and host cell invasion', in D.A.E. Dobbelaere \& D.J. Mckeever (eds.), World class parasites: Theileria, pp. 1-22, Kluwer Academic Publishers, Norwell, Massachusetts, USA

Stoltsz, W.H. \& Dunsterville, M.T., 1992, 'In vitro establishment and cultivation of a Cytauxzoon sp. (Theileria sp.) from a Sable antelope (Hippotragus niger, Harris 1838)', Journal of the South African Veterinary Association 63, 182.

Todd, J.L. \& Wolbach, S.B., 1912, 'Parasitic protozoa from the Gambia', Journal of Medical Research 26, 195-218. PMid:19972051

Wagner, G.G., Jessett, D.M., Brown, C.G.D. \& Radley, D.E., 1975, 'Diminished antibody response to rinderpest vaccination in cattle undergoing experimental East Coast fever', Research in Veterinary Science 19, 290-211.

Walker, A.R., Mckellar, S.B., Bell, L.J. \& Brown, C.G.D., 1979, 'Rapid quantitative assessment of Theileria infection in ticks', Tropical Animal Health and Production 11, 21-26. http://dx.doi.org/10.1007/BF02237760

World Organisation for Animal Health, 2008, 'Theileriosis', in Manual of diagnostic tests and vaccines for terrestrial animals, 6th edn., 2.4.16, pp. 789-804, viewed 23 June 2011, from http://www.oie.int/international-standard-setting/terrestrialmanual/access-online/

Wilson, D.E., Bartsch, R.C., Bigalke, R.D. \& Thomas, S.E., 1974, 'Observations on mortality rates and disease in roan and sable antelope on Nature Reserves in the Transvaal', Southern African Wildlife Management Association Journal 4, 203-206. 